\title{
A TAUBERIAN THEOREM FOR STRONG RIESZ SUMMABILITY
}

\section{PRAMILA SRIVASTAVA}

1.1. Let $\sum_{n=1}^{\infty} a_{n}$ be a given infinite series, and $\lambda_{n}$ a positive monotonic increasing function of $n$, tending to infinity with $n$. For $r>-1$, integral or not, we write

$$
A_{\lambda}^{r}(\omega)=\sum_{\lambda_{n}<\omega}\left(\omega-\lambda_{n}\right)^{r} a_{n} .
$$

When $k \geqq 0$, the series $\sum a_{n}$ is said to be summable $(R, \lambda, k)$ to the value $s$, if

$$
\lim _{x \rightarrow \infty} x^{-k} A_{\lambda}^{k}(x)=s
$$

[3], see also [2]. The series $\sum a_{n}$ is said to be strongly summable $(R, \lambda, k)$, or summable $[R, \lambda, k]$, where $k>0$, if

$$
\int_{\lambda_{0}}^{\omega}\left|x^{-(k-1)} A_{\lambda}^{(k-1)}(x)-s\right| d x=o(\omega),
$$

as $\omega \rightarrow \infty$. If

$$
\sum_{\lambda_{n}<\omega}\left|a_{n} \lambda_{n}\right|=o(\omega)
$$

and $\sum a_{n}$ is convergent, then it is said to be summable $[R, \lambda, 0]$. Replacing $o$ by $O$ and convergence by boundedness in the above, the definitions for boundedness $[R, \lambda, k]$ and $[R, \lambda, 0]$ are obtained [4].

1.2. The first theorem of consistency for strong Riesz summability [4, Theorems 3 and $\left.3^{\prime}\right]$ states that if $r \geqq 0$ and the infinite series $\sum a_{n}$ is summable $[R, \lambda, r]$, then it is summable $[R, \lambda, k]$ when $k>r$. It is trivial that a series summable $[R, \lambda, k]$ may fail to be summable $[R, \lambda, r]$ when $r<k$. A result has already been obtained in the direction of determining some extra condition or conditions which together with summability $[R, \lambda, k]$ may yield summability $[R, \lambda, r]$, $r$ being less than $k[4$, Theorem 8]. The object of this paper is to establish another theorem with reference to the same problem.

It may be mentioned that the theorem of the present paper includes as a particular case a theorem of Winn [5, Theorem $\mathrm{X}]$ for

Received by the editors December 11, 1957 and, in revised form, February 10, 1958.

1 The condition $\mu \leqq k$ of the original lemma is superfluous in view of the extension of the definition for $A_{\lambda}^{k}(\omega)$ to negative $k, k>-1$. 
strong Cesàro summability on account of equivalence of summabilities $[R, n, k]$ and $[C, k][4 ; 1]$.

My warmest thanks are due to Dr. B. N. Prasad for his kind encouragement and helpful guidance.

2.1. Theorem. If $\sum a_{n}$ is summable $(R, \lambda, k)$ for some positive $k$ and is bounded $[R, \lambda, r]$ for some $r \geqq 0$, then it is summable $[R, \lambda, r+\delta]$ whenever $\delta>0$.

2.2. In order to prove the theorem we require the following lemmas.

Lemma 1 [3, p. 27, Lemma 6]. ${ }^{1}$ If $k>0, \mu<1$, then

$$
A_{\lambda}^{k-\mu}(\omega)=\frac{\Gamma(k-\mu+1)}{\Gamma(k+1) \Gamma(1-\mu)} \int_{0}^{\omega} A_{\lambda}^{k-1}(u)(\omega-u)^{-\mu} d u .
$$

LEMma 2 [4, Theorem 1]. If $\sum a_{n}$ is summable (or bounded) $(R, \lambda, k-1)$, then it is summable (or bounded) $[R, \lambda, k]$.

Lemma 3. If $\sum a_{n}$ is summable $[R, \lambda, k+1]$ and bounded $[R, \lambda, k]$, then it is summable $[R, \lambda, k+\delta]$ when $k>0$ and $\delta>0$.

Proof of Lemma 3. We suppose $\delta<1$, as otherwise the result is an immediate consequence of the first theorem of consistency for strong Riesz summability. We may, also, suppose without any loss of generality the sum of the given series to be zero. Hence, being given that

$$
\int_{\lambda_{0}}^{\omega}\left|x^{-(k-1)} A_{\lambda}^{k-1}(x)\right| d x=O(\omega)
$$

and

$$
\int_{\lambda_{0}}^{\omega}\left|x^{-k} A_{\lambda}^{k}(x)\right| d x=o(\omega)
$$

it is to be shown that

$$
\int_{\lambda_{0}}^{\omega}\left|x^{-(k+\delta-1)} A_{\lambda}^{k+\delta-1}(x)\right| d x=o(\omega) .
$$

The conditions (2.1), (2.2) and (2.3) may be replaced by the following alternative forms respectively.

$$
\int_{\lambda_{0}}^{\omega}\left|A_{\lambda}^{k-1}(x)\right| \quad d x=O\left(\omega^{k}\right)
$$




$$
\begin{array}{r}
\int_{\lambda_{0}}^{\omega}\left|A_{\lambda}^{k}(x)\right| d x=o\left(\omega^{k+1}\right), \\
\int_{\lambda_{0}}^{\omega}\left|A_{\lambda}^{k+\delta-1}(x)\right| d x=o\left(\omega^{k+\delta}\right) .
\end{array}
$$

We have, by Lemma 1 ,

$$
\begin{aligned}
C \int_{\lambda_{0}}^{\omega}\left|A_{\lambda}^{k+\delta-1}(x)\right| d x & =\int_{\lambda_{0}}^{\omega}\left|\int_{0}^{x} A_{\lambda}^{k-1}(u)(x-u)^{\delta-1} d u\right| d x \\
& \leq \int_{\lambda_{0}}^{\omega} d x\left|\int_{0}^{x-t}(x-u)^{\delta-1} A_{\lambda}^{k-1}(u) d u\right| \\
& +\int_{\lambda_{0}}^{\omega} d x\left|\int_{x-t}^{x}(x-u)^{\delta-1} A_{\lambda}^{k-1}(u) d u\right| \\
= & I_{1}+I_{2},
\end{aligned}
$$

say, where $C$ is a constant which may be different at different places. Here $t=\xi x$ and $\xi$ is a constant to be determined subsequently. Evidently, it will suffice to show that

and

$$
I_{1}=o\left(\omega^{k+\delta}\right)
$$

$$
I_{2}=o\left(\omega^{k+\delta}\right) .
$$

We first consider $I_{2}$. We have

$$
\begin{aligned}
I_{2} & =\int_{\lambda_{0}}^{\omega} d x\left|\int_{x-t}^{x}(x-u)^{\delta-1} A_{\lambda}^{k-1}(u) d u\right| \\
& \leqq \int_{\lambda_{0}}^{\omega} d x \int_{0}^{t} u^{\delta-1}\left|A_{\lambda}^{k-1}(x-u)\right| d u \\
& =\int_{\lambda_{0}}^{\omega} d x \int_{0}^{\xi x} u^{\delta-1}\left|A_{\lambda}^{k-1}(x-u)\right| d u .
\end{aligned}
$$

By changing the order of integration, we get

$$
\begin{aligned}
I_{2} \leqq & \int_{0}^{\lambda_{0} \xi} u^{\delta-1} d u \int_{\lambda_{0}}^{\omega}\left|A_{\lambda}^{k-1}(x-u)\right| d x \\
& +\int_{\lambda_{0} \xi}^{\omega \xi} u^{\delta-1} d u \int_{u / \xi}^{\omega}\left|A_{\lambda}^{k-1}(x-u)\right| d x \\
= & I_{2,1}+I_{2,2} .
\end{aligned}
$$

say. Now, by $\left(2.1^{\prime}\right)$ 
And

$$
\begin{aligned}
I_{2,1} & =O \int_{0}^{\lambda_{0} \xi} u^{\delta-1}(\omega-u)^{k} d u \\
& =O\left\{\omega^{k}\left(\lambda_{0} \xi\right)^{\delta}\right\}=o\left(\xi^{\delta} \omega^{k+\delta}\right) .
\end{aligned}
$$

$$
\begin{aligned}
I_{2,2} & =O \int_{\lambda_{0} \xi}^{\omega \xi} u^{\delta-1}(\omega-u)^{k} d u+O \int_{\lambda_{0} \xi}^{\omega \xi} u^{k+\delta-1}\left(1-\frac{1}{\xi}\right)^{k} d u \\
& =O\left(\omega^{k+\delta} \xi^{\delta}\right)+O\left(\omega^{k+\delta} \xi^{\delta}\right)=O\left(\omega^{k+\delta} \xi^{\delta}\right) .
\end{aligned}
$$

Supposing $\xi=o(1)$, we obtain

$$
\begin{aligned}
I_{2} & \leqq I_{2,1}+I_{2,2} \\
& =o\left(\omega^{k+\delta}\right),
\end{aligned}
$$

as $\omega \rightarrow \infty$. Also

$$
\begin{aligned}
I_{1} & =\int_{\lambda_{0}}^{\omega} d x\left|\int_{0}^{x-t}(x-u)^{\delta-1} A_{\lambda}^{k-1}(u) d u\right| \\
& \leqq C \int_{\lambda_{0}}^{\omega} d x\left|A_{\lambda}^{k}(x-t)\right| t^{\delta-1}+C \int_{\lambda_{0}}^{\omega} d x\left|\int_{0}^{x-t} A_{\lambda}^{k}(u)(x-u)^{\delta-2} d u\right| \\
& =C I_{1,1}+C I_{1,2},
\end{aligned}
$$

say. By $\left(2.2^{\prime}\right)$, we get

$$
\begin{aligned}
I_{1,1} & =\int_{\lambda_{0}}^{\omega}(x \xi)^{\delta-1}\left|A_{\lambda}^{k}\{x(1-\xi)\}\right| d x \\
& =o(\omega \xi)^{\delta-1} \omega^{k+1}(1-\xi)^{k}+o\left\{\xi^{\delta-1}(1-\xi)^{k} \int_{\lambda_{0}}^{\omega} x^{\delta-2+k+1} d x\right\} \\
& =o\left\{\omega^{k+\delta} \xi^{\delta-1}(1-\xi)^{k}\right\}+o\left\{\xi^{\delta-1}(1-\xi)^{k} \omega^{k+\delta}\right\} \\
& =o\left\{\omega^{k+\delta} \xi^{\delta-1}(1-\xi)^{k}\right\},
\end{aligned}
$$

and

$$
\begin{aligned}
I_{1,2} & \leqq \int_{\lambda_{0}}^{\omega} d x \int_{0}^{x-t}\left|A_{\lambda}^{k}(u)\right|(x-u)^{\delta-2} d u \\
& =\int_{\lambda_{0}}^{\omega} t^{\delta-2} o(x-t)^{k+1} d x \\
& =\int_{\lambda_{0}}^{\omega} x^{\delta-2+k+1} \xi^{\delta-2}(1-\xi)^{k+1} d x \\
& =o\left\{\omega^{k+\delta} \xi^{\delta-2}(1-\xi)^{k+1}\right\} .
\end{aligned}
$$


Hence

$$
I_{1}=O\left(I_{1,1}+I_{1,2}\right)=o\left(\omega^{k+\delta}\right),
$$

by choosing $\xi$ first and then $\omega$. Thus the proof of the lemma is complete.

2.3. Proof of the theorem. By Lemma 2, for the proof of the theorem it is sufficient to show simply that "if $r \geqq 0$ and $\sum a_{n}$ is bounded $[R, \lambda, r]$ and summable $[R, \lambda, k]$, then it is also summable $[R, \lambda, r+\delta]$ where $\delta>0$." We consider first the case $r>0$. If $k \leqq r+1$, the theorem is seen to follow immediately from Lemma 3. And, if $k>r+1$, by the first theorem of consistency $\sum a_{n}$ is bounded $[R, \lambda, k-1]$ and summability $[R, \lambda, k-1+\delta]$ follows from Lemma 3 . By similar repeated applications of Lemma 3, ultimately, summability $[R, \lambda, r+\delta], \delta>0$, of the given series is obtained.

For the case $r=0$, we observe that given a finite number $\delta>0$, we can always write $\delta=\delta_{1}+\delta_{2}$, where both $\delta_{1}$ and $\delta_{2}$ are $>0$. Now, since the series $\sum a_{n}$ is bounded $[R, \lambda, 0]$, it is bounded $\left[R, \lambda, \delta_{1}\right]$ by the first theorem of consistency, and then summability $[R, \lambda$, $\left.\delta_{1}+\delta_{2}\right]$ i.e. summability $[R, \lambda, \delta]$ follows from the part of the theorem already proved. This completes the proof of the theorem.

2.4. The reasoning, given above for the case $r=0$, leads to the following corollary.

Corollary. If $\sum a_{n}$ is bounded $[R, \lambda, k+\delta]$ for every $\delta>0$ and is summable $(R, \lambda, l)$ for some $l$, then it is also summable $[R, \lambda, k+\delta]$.

2.5. Finally it may be pointed out that we can not take $\delta$ to be zero in our theorem. A gegenbeispiel to this effect is provided by the well known alternating series $1-1+1-\cdots$.

This series is bounded $[R, n, 1]$, summable $(R, n, k), k>0$, but not summable $[R, n, 1]$.

\section{REFERENCES}

1. A. V. Boyd and J. M. Hyslop, A definition for strong Rieszian summability and its relationship to strong Cesaro summability, Proc. Glasgow Math. Assoc. vol. 1 (1952) pp. 34-39.

2. K. Chandrasekharan and S. Minakshisundaram, Typical means, Oxford, 1952.

3. G. H. Hardy and M. Riesz, The general theory of Dirichlet's series, Cambridge, 1915.

4. P. Srivastava, On strong Rieszian summability of infinite series, Proc. Nat. Inst. Sci. India vol. 23 A (1957) pp. 58-71.

5. C. E. Winn, On strong summability for any positive order, Math. Z. vol. 37 (1933) pp. $481-492$.

Allahabad University, Allahabad, India 